\title{
FOREWORD
}

\section{Special Section on Knowledge-Based Software Engineering}

The special interest group named Knowledge-Based Software Engineering (SIG-KBSE) was established in 1992 in the Institute of Electronics, Information and Communication Engineers (IEICE).

It is possible to think the software development to be a series of activities to realize human knowledge as an algorithm on the computer. SIG-KBSE offers the opportunities for discussions and the presentations concerning not only a software development method using knowledge engineering but also an actual software development.

SIG-KBSE meeting is held every other month. Besides publishing papers including software engineering and knowledge engineering, SIG-KBSE also supports international conferences. The Joint Conference on Knowledge-Based Software Engineering (JCKBSE) is held every other year in a European country or Russia. The first JCKBSE was held in Pereiaslavl-Zalesskii, Russia in 1994. In that time, JCKBSE stood for Japan-CIS symposium on Knowledge-Based Software Engineering. Then, this symposium expanded to a joint conference of Japan, European countries and Russia. JCKBSE96 was held in Sozopol, Bulgaria in 1996. JCKBSE98 was held in Smolenice, Slovakia in 1998. JCKBSE2000 was held in Brno, Czech in 2000. JCKBSE2002 was held in Maribor, Slovenia in 2002. JCKBSE2004 was held in Protovino, Russia in 2004. JCKBSE2006 was held in Tallinn, Estonia in 2006. The latest JCKBSE was held in Piraeus, Greece in 2008 as the 8th JCKBSE2008.

This section was planned to include papers based on the presentations at JCKBSE2008 and the papers submitted for the CFP.

In preparation of this special section, an Editorial Committee was organized and a CFP was distributed via IEICE transactions. 29 papers were submitted in response to the CFP (20 from Japan, 3 from Korea and from ROC (Taiwan), 2 from Indonesia, 1 from China). After extensive review processes, 11 papers were selected for this special section. This shows the strictness of reviewing processes as well as high quality of accepted papers. The topics covered in this section include Knowledge-based Requirements Engineering, Knowledge-based Software development techniques, Knowledge-based management techniques, and Agent-based system.

Finally, I would like to express my sincere appreciation and gratitude to all the authors for their contributions. Special thanks are due to the following editorial committee members and additional referees for their very considerable efforts to review the submitted papers and their assistance of preparing for this Special Section:

Special Section Editorial Committee Members

Guest Associate Editors-in-Chief:

Taichi Nakamura (Tokyo University of Technology), Takako Nakatani (University of Tsukuba)

Guest Editors:

Shinobu Saito (NTT Data), Hiroya Itoga (Ritsumeikan University)

Guest Associate Editors:

Hirohisa Aman (Ehime University), Koichi Ono (IBM Japan), Kenji Kaijiri (Shinshu University), Atsushi Kanai (Hosei University), Shigeo Kaneda (Doshisha University), Mizue Kayama (Shinshu University), Masakazu Kanbe (NTT Data), Fumihiro Kumeno (Mitsubishi Research Institute), Seiichi Komiya (Shibaura Institute of Technology), Teruo Koyama (National Institute of Informatics), Yasushi Sakurai (NTT), Masaru Nakagawa (Wakayama University), Toyohiko Hirota (Kyushu Sangyo University), Yoshiaki Fukazawa (Waseda University), Naoki Fukuta (Shizuoka University), Rihito Yaegashi (Kagawa University), Takahira Yamaguchi (Keio University), Shuichiro Yamamoto (Nagoya University), Syunsuke Yura (NTT), Hironori Washizaki (Waseda University)

Atsushi Ohnishi, Guest Editor-in-Chief 
Atsushi Ohnishi (Member)

received B. of Engineering, M. of Engineering, and Dr. of Engineering degrees from Kyoto University, Japan in 1979, 1981, and 1988, respectively. He was a Research Associate and an Associate Professor of Kyoto University from 1983 to 1994. Since 1994 he has been a Professor at Department of Computer Science, Ritsumeikan University, Japan. He was a visiting scientist at UC Santa Barbara, U.S.A. from 1990 to 1991 and a visiting scientist at Georgia Institute of Technology, U.S.A. in 2000. His current research interests include requirements engineering, object oriented analysis, and software design techniques. Dr. Ohnishi is a member of IEEE Computer Society, ACM, Information Processing Society (IPS) Japan, and Japan Society for Software Science and Technology (JSSST). He is currently a chairman of SIG Knowledge-Based Software Engineering of IEICE.

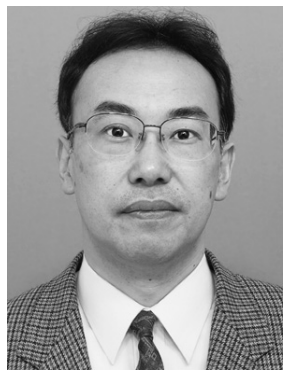

